\title{
A System to Support the Integrated Management of Diagnostic Medical Images
}

\author{
Andrea F. Abate, Rosanna Cassino, Gabriele Sabatino, and Maurizio Tucci \\ Dipartimento di Matematica e Informatica, \\ Università di Salerno,Via Ponte don Melillo, \\ 84084 Fisciano, Salerno - Italy \\ \{abate, rcassino, gsabatino, mtucci\}@unisa.it
}

\begin{abstract}
Information systems are essential tools supporting the management of hospital organizations. The demand for availability and integration of data in this field is more and more increasing, basically for absolving two key issues: collecting and merging all the elementary data available for a patient during the hospitalization process, so that physicians and other operators get all the necessary information they need during the process; planning the development of the diagnostic activities/therapeutics to optimize the process. In this perspective, we present a system that integrates a booking subsystem for hospital specialized treatments booking (CUP), a subsystem for the management of the hospitalizations (including First Aid Departments), a subsystem for filing and reporting clinical images, a subsystem for the analysis of radiological images in a unique management environment. Therefore we describe a complete system for the management of an archive of digital dossiers for the patients of a hospital, where diagnostic imaging is a central issue.
\end{abstract}

\section{Introduction}

The diffusion of informative systems in the field of sanitary firms is very heterogeneous, due to the complex organization of these structures, often articulated in a number of operational units, departments and services, each of them having ample decisional autonomy. Another reason for heterogeneity often lies in the limited budget for the ICT investments that has hindered a univocal approach to the demands of computerizing.

This fragmentation has accordingly determined a proliferation of informative systems, heterogeneous both in architectural terms (hardware platforms, software technologies and nets), and in applicable and functional terms (interfaces, instructions and processes). So many separate "islands" have been created that they do not even communicate among them. The need of integration of systems and information is now becoming more apparent to the medical and nursery staffs themselves, as they are experimenting increasing difficulties in matching today's management objectives using old technologies and traditional tools [10].

The role of the Departments of Diagnostic Radiology is particularly relevant in the development of management systems supporting the diagnostics processes, as the Ra- 
diological Information Systems (RIS) are assuming a leading identity within the Hospital Information Systems in the definition of requirements and objectives and in the evaluation of innovative technical solutions.

Typical database solutions to manage patient related data (acceptance, scheduling, filing, statistics) are usually implemented in the existing RIS systems, but tools for the integrated management of diagnostic imaging in PACS systems are often still lacking, so that many Radiology Departments are still storing physical diagnostic images although more efficient and less expensive digital storing solutions are now available and in many cases allowed by specific laws and regulations [12].

In this perspective, in this paper we present an integrated system to manage both the administrative information of the patients and the achievement and analysis of the related medical images. This work was developed within a project founded by the Italian Minister of Health Care and developed by the "Girolamo Orlando" Foundation of Pescopagano in collaboration with the Department of Mathematics and Computer Science at Salerno University. The goal of this project was the functional integration of a traditional management system for hospital treatment booking (CUP), hospital admission management and medical reporting with a diagnostic imaging system $[1,2]$.

The paper is organized as follow. Section 2 describes the proposed system architecture underlying the characteristics of the integration between the several involved systems. In section 3 we present the implementation characteristics of the integrated environment. Finally, in section 4 we show some conclusion and any further work.

\section{System Architecture}

In this paper we present a case study related to the development of an integrated system to manage the administrative information of the patients and the achievement and analysis of the related medical images. The developed prototype intends a series of objective: produce new tools to aid the physician for the analysis of the images and for the formulation of the diagnosis through the native comparison of images to known diagnosis; create a given basic representative of the expertise, that is a Knowledge Basic Management System of the diagnosis, of the treatment protocols, etc.; provide a support to the didactic; make available the expertise in the net, create a database for possible recourses legal physician. In the integration process between the several applications - all object oriented - the processes of interaction and exchange of the data has stayed standardized and conformed to the more recent specifics in terms of safety and of efficiency. Therefore, all the exchanges occur by web services, e the process to receive or send the data by XML/HL7 (v.2.3.1) [9], in domain ports logic, respectively (applicative, delegate). Such specific allows using only the web type net protocol (http, https) and then an impact reduced on the configuration of possible net devices. Particularly, the communication foresees that the receiving entity exposes a web services, called from the transmitting entity for the dispatch of the data.

Figure 1 presents a general description of the flows that interest all the evolution phases in which a contact could be generally dismountable. 


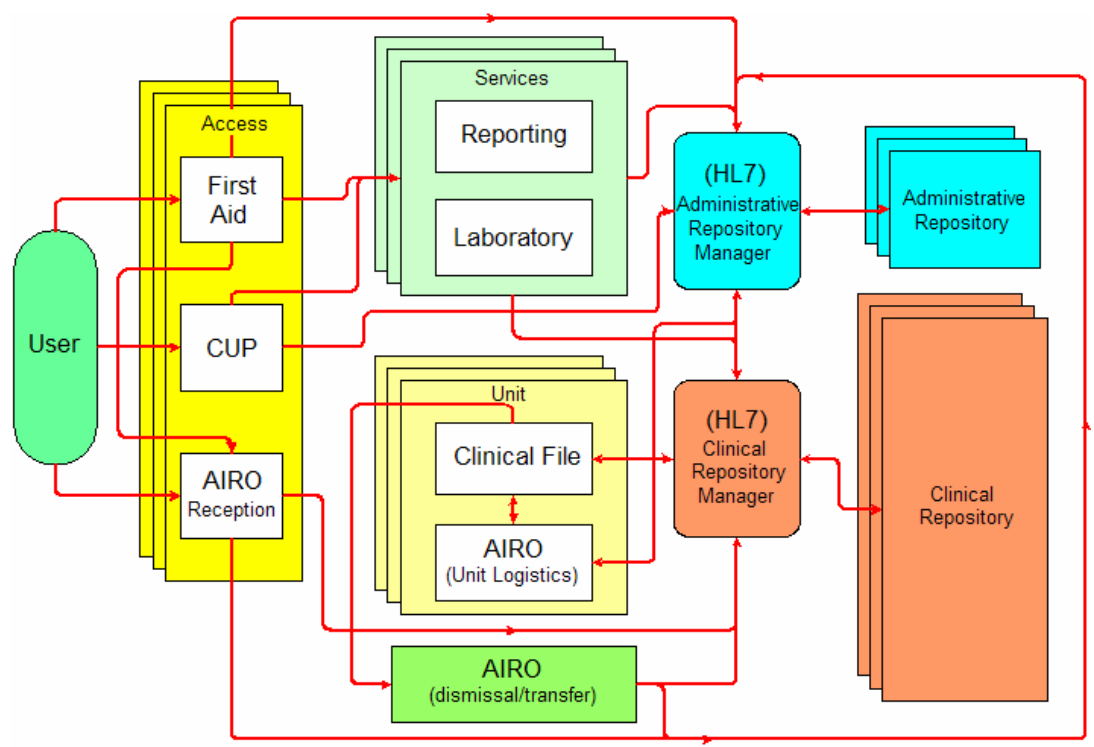

Fig. 1. Flows of the information of a patient to contact with a sanitary structure

The informative sanitary system taken to reference is based essentially on the integration of the functionalities which a whole of procedures each of which appointed to the management of specific appearances of the system offers. All the procedures refer to the "Administrative Repository" and to the "Clinical Repository" database. The "Administrative Repository" contains the registry information of the patients that have had any contact with the sanitary structures. The "Clinical Repository" contains the digital clinical files, the reports and the links to the medical images that are in the database of the servers where such pictures are achieved and related to the other information of the patients. The new reporting environment allows the inside interaction with the CUP processes (related to the bookings of specialized performances) with the systems for the management of the activities of First Aid and of the Departments (AIRO - hospitalization informative area) and finally with all the tools to analyze and process the clinical images.

The problems related to the management of the clinical images could be outlined through a process of analysis and diagnosis composed of four separate phases: acquisition and elaboration of the images; management of the data and of the images; diagnosis formulation; classification and filing of the information. Each phase is not isolated but is support and verify for the others. Figure 2 shows the applicative process model that describes the potentialities of the proposed prototype in the complex. The extreme versatility of the prototype allows wide integration with all the other present systems in the hospital structures beyond that the possibility from part of all the authenticated user from the system to access to a large field information, by means of a simple internet connection, service staying the presence of the tricks to safeguard the sensitive data and to respect the privacy. 


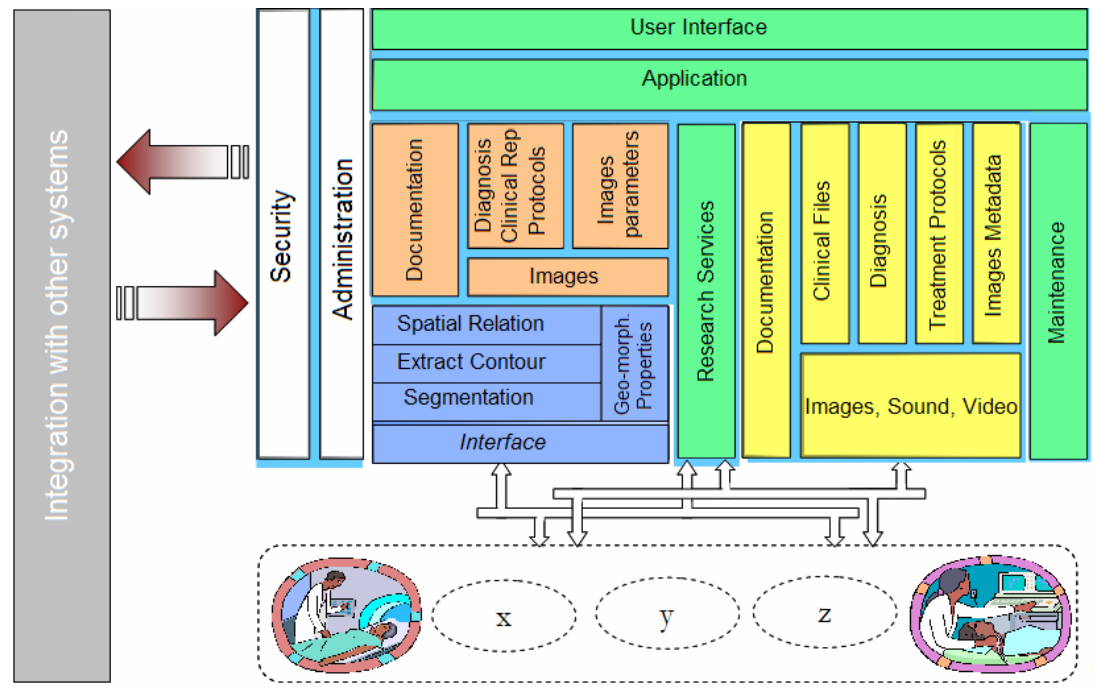

Fig. 2. The applicative process model

The clinical images elaboration phases, after they have stayed achieved according to DICOM 3 protocol [11] are:

- segmentation,

- extract contour,

- evaluation of the geo-morphological properties,

- spatial relations computing.

If the image contains some abnormality whose geo-morphological characteristics are automatically extracted within the corresponding spatial relations. In this matter, the analysis of a pathology proceeds with the calculus of its geo-morphological characteristics and on the basis of the spatial arrangement of these last ones. All such tasks are performed using different algorithms already available in the literature. In detail, the analysis of a clinical image segments the images, underlines the contours of the individualized abnormality (segmentation [8] and edge detection phases [6]), computes the geo-morphological parameters (information related to the density of the analyzed object, asymmetry, orientation, spreadness, uniformity, convexity $[3,4,5]$ ) and, by means of the spatial relationship between the objects (particularly the representative objects of the abnormality and the surrounding anatomical components) describes the virtual image [7] related to the examined image. Then, after the clinical images of a patient are achieved, these will be analyzed inside the environment of the described system. The physician will mail the criterions of search and visually formulate queries that extract from the database all the images similar to the analyzed images on the base of the matching of the geo-morphological parameters understood in a determined range of values of reference. The physician verifies the drawn out contents and analyzes the agreement with regards to his interest. In the case the system has extrapolated objects coherent with the abnormality to diagnose, he could recall all the documentation correlated to the image (administrative and clinical data and above all the reports already issued), so to be supported in the new diagnosis phase. 
All useful information to the updating of the clinical file of the patient are organized on the base to their content for then have filed correctly and represented graphically. The classified data are: the administration documentation, the patient personal data, the diagnosis, the treatment protocols, the clinical files, the images parameters, (see figure 2). The categorized operations allow to feed the database and, on the base of the instruction of the user about the informative grade of the sample document to which he has had access, it is possible optimize the documents presentation. Thanks to the presence of a thematic engine, through the web, it is possible to agree to all the information of the database. It represents a real "Knowledge Base Management System", useful for the physician, furnishing aid to the analysis of the images and to the diagnosis formulation, for the Hospital Institutes present on the territory, allowing the expertise in the net, and for the Universities Institute, constituting a formidable information field supporting didactic.

\section{System Implementation}

As stated before, the aim of this work was integration between a management system for patient administrative information and a system achieving, analyzing and processing related medical images.

The first system is implemented in .NET environment using Microsoft SQL Server whereas the second uses Java and an Oracle database.

As a first step, we planned to use web services technology as a standard for interoperability between different environments. After a services oriented analysis, we pointed out that physicians are less comfortable with different windows on desktop especially during diagnosis report so we decided porting all systems in .NET environment using SQL Server as DBMS. We used J\# to migrate Java code in .NET environment.

Inside the subsystem devoted to diagnostic imaging are implemented the processes of segmentation and edge detection of the individuated pathology described in previous section (Fig. 3).

Once the contour of the abnormality located in the examined radiological image is extracted, the analysis of pathology proceeds with the calculus of its geomorphological characteristics. This information will be used for querying the database relatively to the similarity retrieval for geo-morphological characteristics of other images previously examined.

The proposed Visual Data Base Management System is based on integration of icon based methodology used in GIS systems with similarity retrieval techniques in the field of diagnostic imaging systems. This helps the diagnosis process of physicians that could use a retrieval technique in order to access other documents into the Database. There is also the possibility to express a diagnosis using a template including all information related to medical examination and annotations if necessary.

Such a process could be used even if physician is interested in analyzing an examination or comparing with similar ones. 
From such point of view, the whole process is a completion of applicationfunctional aspect that allows the systems to manage the digital dossier of a patient and his clinical history.

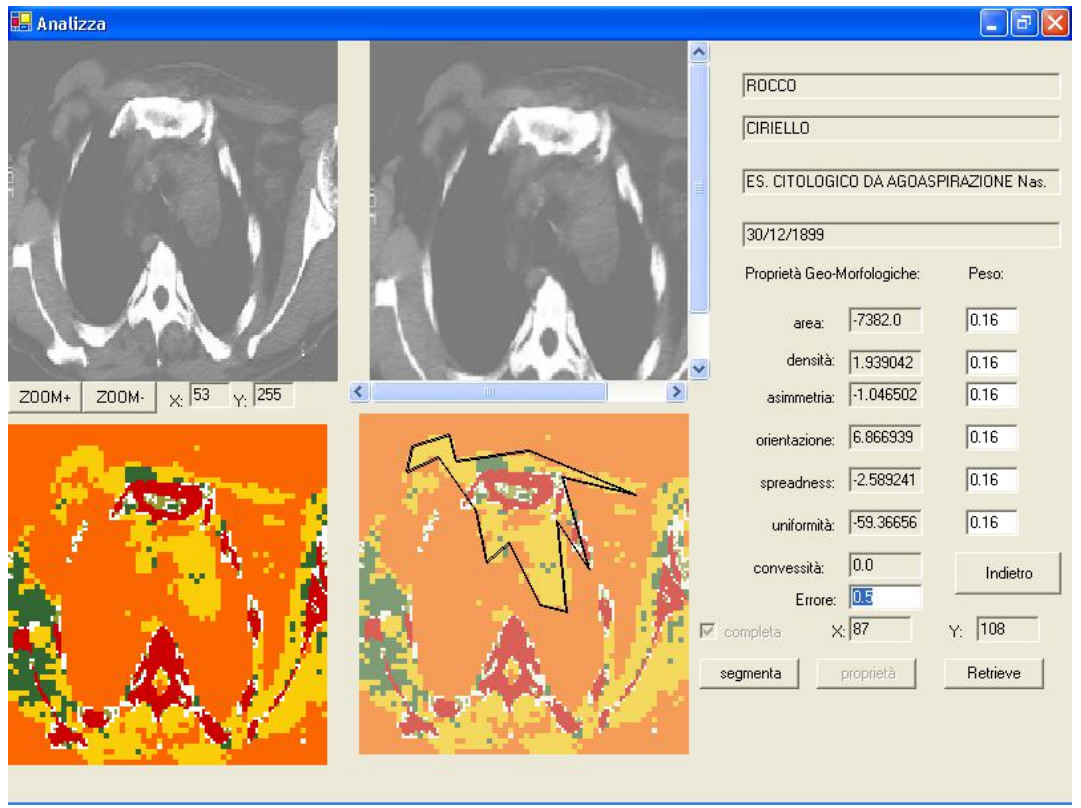

Fig. 3. Segmentation and geo-morphological properties of an image

\section{Conclusion and Further Work}

The system illustrated in the previous sections supports hospital centers in the integrated management of every patient-related information available during a treatment plan, including diagnostic imaging. The main feature of this application is flexibility, which lies in its ability in customizing the user interface for any particular department's needs.

Currently, the system has been proposed to different sanitary firms like the hospital "S. Carlo" in Potenza (Italy) and the Azienda Sanitaria Locale (ASL) n. 2 in Venosa, Potenza (Italy). Future work will focus on using the results of the present case study evaluation to predict the innovation impact of introducing an integrated RIS/PACS system in these real hospital contexts. Preliminary tests on the presented application have indeed shown that the availability of integrated information effectively enhances a RIS/PACS system's support of diagnostic activities.

Further evolution of this work will consider new image processing functions to manipulate and manage diagnostic images, especially to provide physicians with 3D-based visualization for image analysis and retrieval. 


\section{References}

[1] Abate A. F., Cassino R. and Tucci M.: An Integrated Environment for Control and Management of Pictorial Information Systems. Proceeding of First International Workshop "Image 2003: Learning, Understanding, Information Retrieval, Medical", World Scientific Press, Vol. 15, (2003) 123-131

[2] Abate A. F., Nappi M., Tortora G., Tucci M.: IME: An Image Management Environment with Content-based Access, Image and Vision Computing, Elsevier Science, Vol. 17, (1999) 967-980

[3] Bryant A.: Recognizing Shapes in Planar Binary Images. Pattern Recognition, Vol. 22, (1989) 155-164

[4] Dunham J. G.: Optimum Uniform Piece Linear Approximation of Planar Curves. IEEE Transaction on Pattern Analysis and Machine Intelligence, PAMI, Vol. 8, n. 1, (1986)

[5] Gritzali F., Papakonstantinou G.: A Fast Piece Linear Approximation Algorithm, Signal Processing, Vol. 5, (1983) 221-227

[6] Papakonstantinou G..: Optimal Polygonal Approximation of Digital Curves. Signal Processing, Vol. 8, (1985) 131-135

[7] Petraglia G., Sebillo M., Tucci M., Tortora G.: Virtual Images for Similarity Retrieval in Image Databases, IEEE Transactions on Knowledge and Data Engineering, Vol. 13, n. 6, (2001), 951-967

[8] Vitulano S., Di Ruberto C., Nappi M.: Different methods to segment biomedical images, Pattern Recognition Letters, Vol. 18, (1997)

[9] http://www.hl7.org

[10] http://www.microsoft.com/italy/biztalk/

[11] http://medical.nema.org/

[12] http://www.teinos.com/ita/ris_pacs_it.html 